\title{
The Multisensory Environment (MSE) in Dementia Care: Examining Its Role and Quality From a User Perspective
}

Health Environments Research \& Design Journal 2017, Vol. 10(5) 39-5I (C) The Author(s) 2016 Reprints and permission: sagepub.com/journalsPermissions.nav DOI: $10.1177 / 1937586716683508$ journals.sagepub.com/home/herd

(SAGE

\author{
Lesley Collier, PhD, MSc, DipCOT', \\ and Anke Jakob, PhD, MA, DipDesign ${ }^{2}$
}

\begin{abstract}
Background: Multisensory environments (MSEs) for people with dementia have been available over 20 years but are used in an ad hoc manner using an eclectic range of equipment. Care homes have endeavored to utilize this approach but have struggled to find a design and approach that works for this setting. Aims: Study aims were to appraise the evolving concept of MSEs from a user perspective, to study the aesthetic and functional qualities, to identify barriers to staff engagement with a sensory environment approach, and to identify design criteria to improve the potential of MSE for people with dementia. Methods: Data were collected from 16 care homes with experience of MSE using ethnographic methods, incorporating semi-structured interviews, and observations of MSE design. Analysis was undertaken using descriptive statistics and thematic analysis. Results: Observations revealed equipment that predominantly stimulated vision and touch. Thematic analysis of the semistructured interviews revealed six themes: not knowing what to do in the room, good for people in the later stages of the disease, reduces anxiety, it's a good activity, design and setting up of the space, and including relatives and care staff. Conclusion: Few MSEs in care homes are designed to meet needs of people with dementia, and staff receive little training in how to facilitate sessions. As such, MSEs are often underused despite perceived benefits. Results of this study have been used to identify the design principles that have been reviewed by relevant stakeholders.
\end{abstract}

\section{Keywords}

dementia, multisensory environments, design, Snoezelen, activity

Sensory rooms, multisensory environments (MSE), and Snoezelen environments are terms used to describe a multisensory space that can be used to provide sensory stimulation or to reduce sensory demand in order to increase engagement and reduce behaviors perceived as challenging (Collier, McPherson, Ellis-Hill, Staal, \& Bucks, 2010; Staal, Pinkney, \& Roane,

\footnotetext{
' Faculty of Health Sciences, University of Southampton, Southampton, United Kingdom

${ }^{2}$ Faculty of Art, Design and Architecture, Kingston University, London, United Kingdom

Corresponding Author:

Lesley Collier, PhD, MSc, DipCOT, Faculty of Health Sciences, University of Southampton, Southampton, SOI7 IBJ, United Kingdom.

Email: ljp@soton.ac.uk
} 


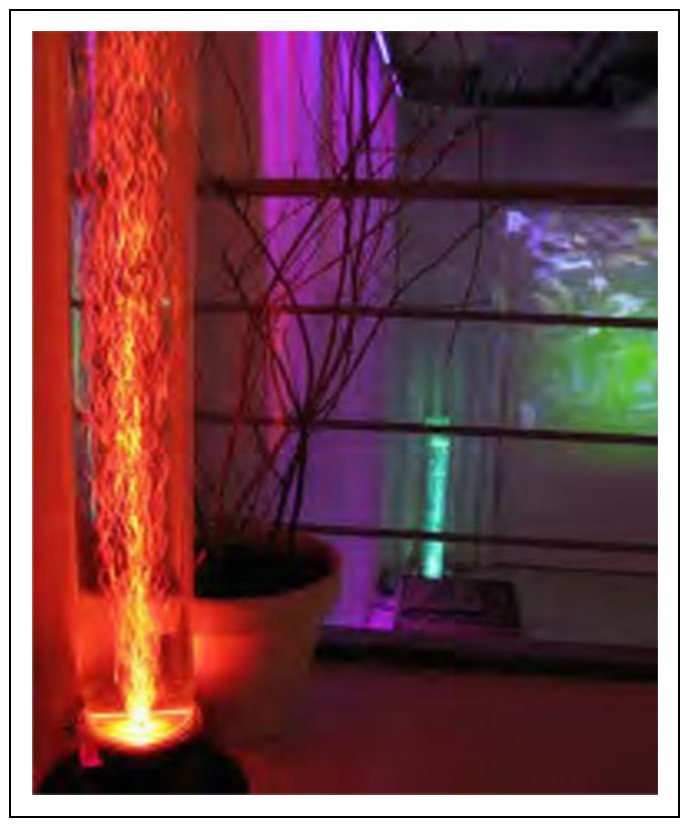

Figure I. Example of a sensory space.

2003). These sensory environments incorporate equipment that stimulate the senses of sight, sound, touch, smell, and movement. Equipment used to stimulate these senses include bubble tubes, vibrating cushions, aroma diffusers, and music. An example of a sensory space is illustrated in Figure 1. These sensory environments have been used for people with dementia with some success for over 20 years (Baker, Dowling, Wareing, Dawson, \& Assey, 1997; Moffat et al., 1993); however, their use within care homes has been inconsistent. Care homes often indicate lack of space or lack of evidence to support implementing a sensory environment, despite a growing body of evidence for their use in reducing agitation and improving functional performance (Collier et al., 2010; Maseda et al., 2014; RileyDoucet \& Dunn, 2013; StrØmb, Ytrehus, \& Ellen-Karine, 2016). Further, in cases where sensory rooms have been installed, it has been reported that they often fail to succeed because of inadequate or poor design and haphazard arrangements (Dalke \& Corso, 2010).

\section{These sensory environments incorporate equipment that stimulate the senses of sight, sound, touch, smell, and movement.}

The concept of Snoezelen was first established in the Netherlands, at the De Hartenburg Institute, where it was constructed as an activity to engage people with severe learning disabilities who were unable to participate in more conventional occupations (Hulsegge \& Verheul, 1987). It was developed from a leisure-based activity to become a therapeutic intervention for people with cognitive impairment across the life span (Baker et al., 1997; Hogg, Cavet, Lambe, \& Smeddle, 2001). Subsequent research using the Snoezelen/multisensory approach for people with dementia has been mixed, due to identified methodological weaknesses. However, recent systematic reviews (Livingston et al., 2014; Sanchez, Millan-Calenti, Lorenzo-Lopez, \& Maseda, 2013) suggest there is some evidence that sensory approaches have the potential to reduce agitation and improve mood in people with dementia; improve occupational engagement (Collier et al., 2010); manage perception of pain (Scholfield, 1996); and increase engagement and social interaction in terms of well-being, quality of life, and quality of care (Lykkeslet, Gjengedal, Skrondal, \& May-Britt, 2014; StrØmb et al., 2016; van Weert, van Dulmen, Spreeuwenberg, Bensing, \& Ribbe, 2005). Nevertheless, further research in this field is needed to obtain more conclusive evidence. To help support the available limited evidence, theoretical perspectives from neuroscience and humanistic philosophy have been used to support the notion of sensory stimulation to enable an individual to interact effectively in their environment (Ayres, 1972; Kovach, 2000). These perspectives describe the interaction of the sensory environment on the individual and subsequent behavioral response from a functional perspective such as the effect of sensory demand on the ability to dual task or carry out a sequence of activities (Kovach, 2000; Schaaf \& Miller, 2005). Consequently, the use of multisensory approaches to manage comorbid agitation and other noncognitive symptoms using a personcentered approach has been documented in the National Institute for Health and Care Excellence (NICE) guidelines: Dementia: Supporting People With Dementia and Their Carers in Health and Social Care. These guidelines are recommendations for treatment and care of 
people with dementia in England and Wales. Despite evidence suggesting that sensory approaches and MSEs such as Snoezelen are of benefit, their use in care home settings is often limited.

Inactivity is often seen as a common feature for people with dementia living in care homes, with participation decreasing as the disease worsens (Leone, Deudon, Piano, Robert, \& Dechamps, 2012). Where activities do occur, they are often constructed around occupations that staff select and that require cognitive functioning such as memory, problem-solving abilities, and attention. Given the difficulties people with dementia have with areas of cognition such as those mentioned above, activities offered by care staff often fail to engage individuals as they fail to optimize remaining abilities and interests (Kolanowski, Litaker, \& Buettner, 2005). Care homes publicize the breadth of activities on offer rather than the success of activities offered. In an attempt to provide activity that might be better suited to the cognitive ability of residents, many care homes invested in sensory equipment and sensory environments such as Snoezelen.

Despite the potential offered by sensory activities, over time, these approaches have been shelved or incorporated into general living areas as staff have had mixed success in using them (Anderson, Bird, MacPherson, McDonough, \& Davis, 2011). Previous surveys of sensory environments in nursing homes have found them to be used in an ad hoc manner using an eclectic range of equipment with staff receiving little formal training (Bauer, Rayner, Koch, \& Chenco, 2012).

\section{Previous surveys of sensory environments in nursing homes have found them to be used in an ad hoc manner using an eclectic range of equipment with staff receiving little formal training.}

Therefore, this study endeavored to identify what equipment care homes were using in their sensory spaces and staff response to using these room designs. The study aims were:

- to study the sensory elements (aesthetic and functional qualities) of existing multisensory room design criteria available in care homes for people with dementia and how they are used;

- to critically evaluate the findings, highlighting barriers to using multisensory equipment identified by staff as well as examples of good practice; and

- to identify room design criteria to improve/ maximize the use of MSE approaches for people with dementia living in care home settings.

\section{Method}

\section{Ethics}

Approval from Kinston University Ethics committee was sought and gained prior to the start of the study.

\section{Procedure}

A purposeful sample of 16 care homes within the south of England with existing/previously existing/planned sensory space or MSE rooms were identified. Inclusion criteria included private and social services provision care homes in the South of England who take residents with a diagnosis of dementia. Care staff within these homes were approached with details of the study using a participant information sheet. Those who were willing to participate met with the research team at a mutually convenient time and completed a written consent form.

\section{Data Collection}

Data were collected by the research team using ethnographic methods incorporating semistructured interviews with care staff to describe sensory facilities available, how the sensory spaces were used, and the experiences of staff in using existing MSE rooms. For those homes which no longer had MSE rooms $(n=5)$, staff were asked about their previous experiences of how sensory spaces were used. Semi-structured, in-depth, face-to-face interviews were undertaken at a mutually convenient time and took approximately $30 \mathrm{~min}$ to complete to elicit detailed and 
descriptive data. The semi-structured interview utilized an interview template including a number of open-ended questions and prompts. The development of the questionnaire was guided by current literature in the field (Fleming \& Bennett, 2015; Garre-Olmo et al., 2012; Moore et al., 2011) and was peer reviewed by experienced researchers at the University of Southampton prior to being submitted to the relevant ethics committee. The interview schedule provided a framework of subject areas to be considered during the interview and ensured consistency of issues explored with all participants (Patton, 2002). The questionnaire also included demographic information of participants including current caring position, information and training received regarding the use of multisensory approaches, and the suitability of multisensory equipment available in the care home.

Observations were made by the research team of the MSE design in each care home including what sensory equipment and items were available under each of the sensory domains (sight, sound, touch, taste, smell, and movement), how the space was set up, how facilities were integrated into the general living environment, and examples of successful practice undertaken by staff. A similar template to the questionnaire was developed to ensure parity of observations across the different care home settings. The template included layout of the setting, details of sensory equipment available, job description details of the staff involved in sensory activity, and details of any activity frameworks being used to guide activity. The observation template was also guided by current literature in the field. Extraneous information or observations made while completing the questionnaire with each individual participant were recorded in a fieldwork diary and were considered postanalysis. Anonymity was achieved with pseudonyms and removal of personal information from the data. On completion of the data collection, themes identified were reviewed by a steering group comprising of designers, healthcare professionals, and home care organizations. These themes were then developed into design principles that have been published separately to this article.

\section{Analysis}

Data from the semi-structured interviews were transcribed and analyzed using a thematic analysis framework as described by Braun and Clarke (2006). This framework provides a systematic guide to analysis using six stages of thematic analysis. The stages involve (1) familiarizing yourself with the data, (2) generating initial codes, (3) identifying themes, (4) reviewing identified themes, (5) defining and naming identified themes, and (6) final analysis and production of the report. Each of these six steps was undertaken in a systematic manner as described below. Questionnaires containing structured information and free text were scrutinized by two researchers independently and independent initial thoughts noted. Initial codes were created based on an inductive approach from the questionnaire data. From these codes, potential themes were agreed upon by the two researchers and data surrounding these themes gathered together. Each theme reflected the overall story described by participants. Saturation was achieved after reviewing 30 questionnaires. The final categories were reviewed by an independent reviewer before final theme names were identified. Rigor of the analysis was maintained by triangulation between the two researchers and peer reviewing of codes and themes in order to promote the credibility of the emerging themes (Polit \& Beck, 2013). Participants and the steering group were also invited to review the findings to ensure their views had been reflected accurately.

Given observations were made using a structured checklist template, analysis of the observations was undertaken using descriptive statistics. Notably, numbers and type of equipment available, staff involved in sensory activity, and details of activity frameworks used to guide activity. Interobserver agreement was ensured by checking the observations undertaken with the member of staff in charge of the home on the day. This ensured the data accurately reflected the equipment available, staff occupation, and activity guidance used.

\section{Results}

The results from this study revealed a number of issues. Firstly, staff acknowledged training was 
Table I. Details of Care Homes.

\begin{tabular}{lr}
\hline Demographics & $N$ \\
\hline Care homes recruited & 16 \\
Care staff interviewed & 32 \\
Private care homes & 14 \\
Social services care homes & 2 \\
Care homes with access to OT for sensory advice & 7 \\
Care homes with activity co-ordinator & 16 \\
Care homes with MSE or sensory space & 11 \\
\hline
\end{tabular}

Note. OT = Occupational Therapy; MSE = multisensory environment.

available but was limited. They expressed a desire for training that focused on more personalcentered sensory approaches. Secondly, there was a recognition that Snoezelen environment and MSE appeared to provide most benefit to residents at the later stages of the disease. Staff believed a sensory approach had the potential to improve well-being for residents in the later stages of the disease as well as for family members. Finally, equipment design needed to be more age appropriate with a stronger focus on reminiscence and familiar objects. The results also revealed there was an overreliance on visual equipment. These findings will be explored in more detail.

Details of the care homes and care staff who participated in the study are presented in Table 1. Care staff interviewed included care home managers, care staff, nursing staff (both qualified and unqualified), and activity co-ordinators who had an interest in or facilitated sensory activities in the care home setting. Responses for individual professional groups were not analyzed, as a response from the whole team was more desirable.

Training in the use of sensory activities and the MSE for carers was mixed (see Table 2). Specialist training was provided by equipment suppliers or outside training providers and focused largely on how to use the equipment. In-house training was provided by staff with an interest in sensory activity but not necessarily formal training. This training focused more on sharing successful experiences.

\section{Training in the use of sensory activities and the MSE for carers was mixed.}

Table 2. Training Opportunities.

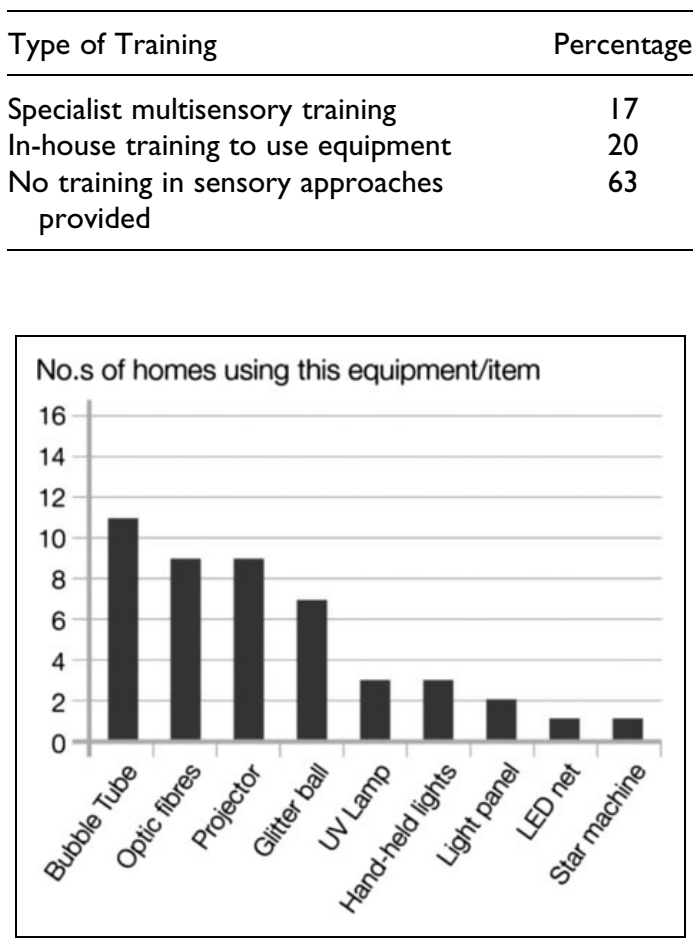

Figure 2. Visual stimulation.

Multisensory equipment available in sensory spaces was variable, with most rooms featuring predominantly visual $(32 \%)$ and tactile stimulation (24\%) such as bubble tubes and optic fibers. Types of equipment available in the sensory spaces are reported in Figures 2-7.

It is notable that very few spaces featured materials and equipment to stimulate proprioception, vestibular system, or taste (gustatory).

Following thematic analysis, data revealed that participants were unsure of how to use the multisensory equipment or set up a sensory space, but they were aware that the approach had potential to engage some of their residents who found it difficult to engage in more conventional activity. Six main themes emerged from this analysis.

Not knowing what to do in the room. This theme focused on what should happen in a sensory space or MSE. Staff liked the idea of the room but were unsure of what they were supposed to do in the 


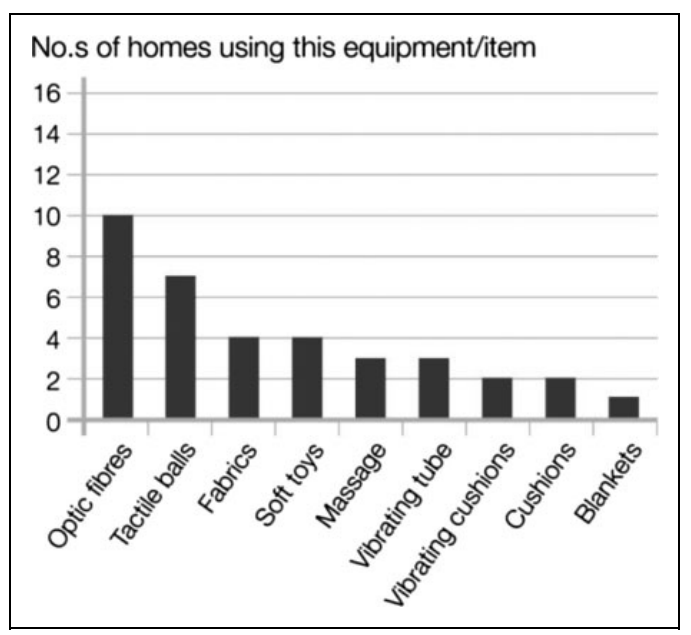

Figure 3. Tactile stimulation.

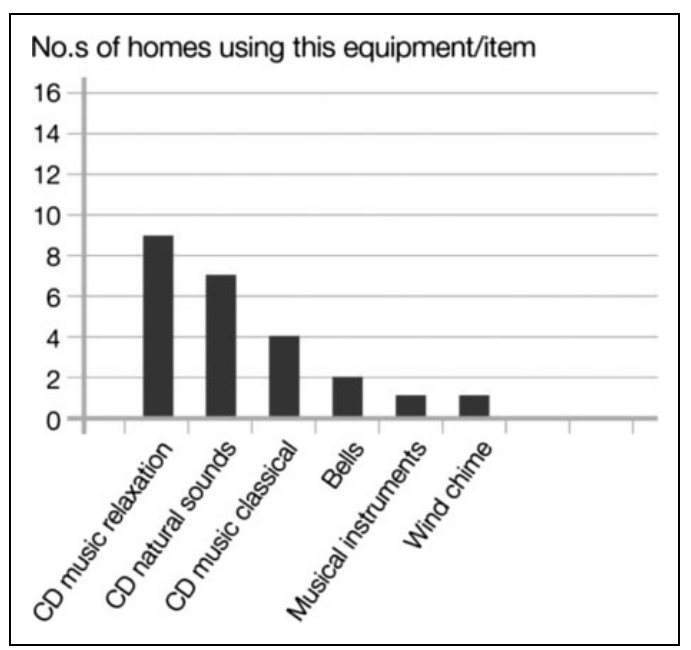

Figure 4. Auditory stimulation.

space or how to use the equipment. There was recognition that they needed help in constructing a useful sensory session with guidance on how to use the equipment and how to grade it for people with different abilities.

I really enjoy doing activity but don't know what to do in the MSE. (P002)

Training that was available focused on the mechanics of operating the equipment rather than how to assess an individual to identify their sensory needs and set up a suitable sensory session. Staff were able to identify their limitations in using this approach and actively requested further training.

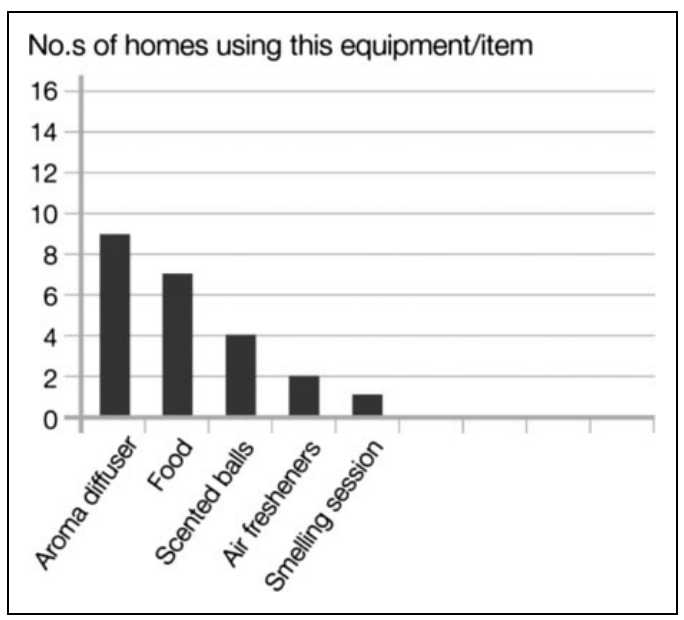

Figure 5. Olfactory stimulation.

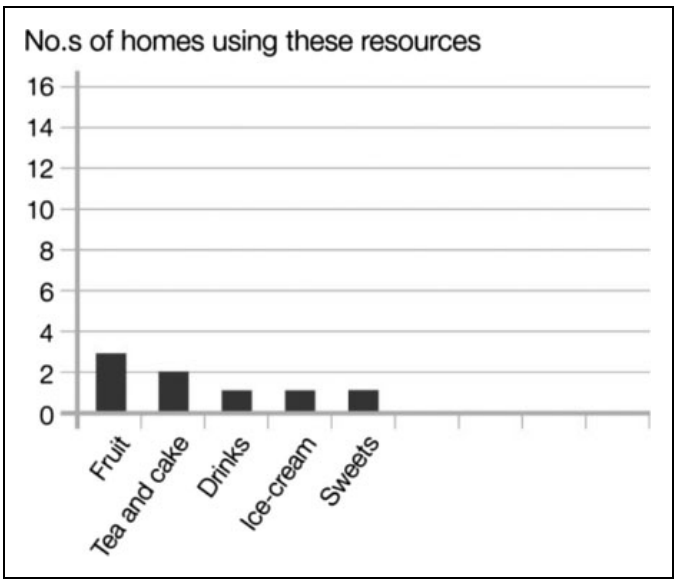

Figure 6. Gustatory stimulation.

We need more training. (P007)

Really want to know more about the Sensory Room. (P018)

There was recognition that they needed help in constructing a useful sensory session with guidance on how to use the equipment and how to grade it for people with different abilities.

Observation notes supported these comments, as staff would often run a sensory session with residents of mixed abilities and different levels of dementia. As a result, some residents would either fall asleep or leave the sensory space, as they were unable to engage with the activity. 


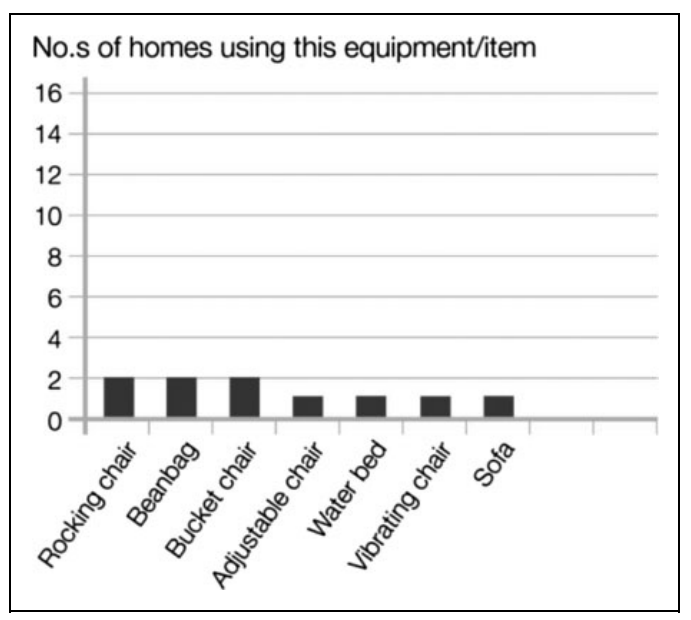

Figure 7. Vestibular/proprioceptive stimulation.

\section{Good for People in the Later Stages of the}

Disease. This theme was drawn from comments about activity for people in the later stages of the disease process. Staff felt the environment was good for people in the later stages of the disease, particularly those who could not participate in more conventional activities. Staff were keen they offered a good level of care that met the person's needs for those at the later stages of the disease and felt the sensory environment was a good place to do this. There was an acknowledgment that this approach was more holistic, focusing on well-being and engagement.

It is good for people who cannot stimulate themselves.... the MSE is good for late stage [dementia] when they don't talk anymore. (P002) it provides a more holistic model of care. (P021) encouraging staff to do more activities not just care. (P007)

the more advanced the dementia, the more intimate the [sensory] interaction needs to be. (P002)

\section{Staff were keen they offered a good level of care that met the person's needs for those at the later stages of the disease and felt the sensory environment was a good place to do this.}

Some participants felt the sensory approach was highly successful and was enjoyed by many of their residents who were no longer able to engage in conventional activities due to the severity of their disease. Those who used the Pool Activity Level (PAL) Instrument for Occupational Profiling-Planning and implementing sensory interventions (Pool, 2012) criteria, which provides guidance on how to run a sensory session (Collier, 2012), described how those at the sensory level benefited most from the sensory space.

For some [residents] the MSE is the only activity they like. (P018)

Residents in sensory state of dementia should be using the room daily, because this would be their activity. (P021)

\section{Reduces Anxiety}

This theme reflected the effect of the MSE on mood and behavior. Staff noted that people became a lot calmer in the room. They perceived this to be a good thing. Staff also felt the resident falling asleep in the room was good, although some felt this behavior could have indicated boredom. Staff thought the space was only there to relax not to stimulate.

\section{It's a very calming atmosphere. (P008) \\ makes the residents more relaxed and calmer. (P023)}

The questionnaires revealed that care staff believed that their role was to provide a calm environment for their residents and that the sensory environment was an alternative to medication.

Reduces the time when we have to give medication, bring the residents to SR first to calm them down. (P008)

Quiet environment makes them feel settled, less agitated, helps with aggression, calms them down, for people with severe dementia and bed-bound. (P007)

\section{It's a Good Activity for Staff and Residents, We Both Benefit}

This theme related to how the activity made the staff member feel. Many staff emphasized that 
this activity was reasonably easy to implement and they also gained benefit from the sensory activity both in feeling more relaxed themselves and in enhancing their relationship with the resident. Both managers and care staff recognized that there was a relationship between the wellbeing of the staff and the quality of subsequent interactions with residents.

It gives me real pleasure to see a resident taking part in something...(P016)

It makes work more interesting, if you can give residents some enjoyment then you feel fulfilled as well. (P013)

Happy residents—happy staff. (P007)

You make somebody's day ... (P018)

The sensory spaces appeared to empower many staff, giving them the sense that they could contribute to a resident's well-being. Staff also reflected on their own participation in their relationship with the resident and were keen to repeat the experience. ( $\mathrm{P} 007)$

If residents can be helped through SR [sensory room], that helps staff as well.... if residents are happy staff is happy as well. (P007)

It is a place where I feel I can help the residents. (P018)

Both managers and care staff recognized that there was a relationship between the well-being of the staff and the quality of subsequent interactions with residents.

\section{Design and Set Up of Space}

This theme summarized thoughts about equipment design. Staff reported frustration at equipment that did not work, was too "childish," or did not fit with the general furnishings of the home. There was a feeling that suppliers did not produce suitable equipment for older people and that the environment should include more familiar or reminiscent sensory items as well as abstract equipment. Staff felt that equipment catalogues did not give them clear advice on what was suitable. Many staff commented on the need to make equipment accessible and recognizable. The inclusion of everyday items was also considered desirable.
Making it more like home with familiar things. (P011)

More natural things need to be included. (P029)

More tactile stimulation and reminiscence stimulation is needed in the room. (P021)

There was a feeling that suppliers did not produce suitable equipment for older people and that the environment should include more familiar or reminiscent sensory items as well as abstract equipment.

Staff also recognized that the sensory approach needed to be extended beyond the sensory space into other living areas.

Make links with the environment outside the Sensory Room. (P019)

Design of the sensory space was commented on by many staff. They reflected on the issues of accessibility and suitability of furnishings. There was a recognition that residents needed to feel comfortable and secure in order to want to go into the sensory space. By making the space feel less clinical seemed to be a solution offered up by many participants.

The room could be used more often if it was set up properly_different feelings of chairs, things to cuddle - a warm, soft and safe, more integrated space. (P029)

environment is very clinical looking at the moment... more homely feel would be better. (P011)

\section{Including Relatives and Care Staff}

This theme was about the need to involve more people. Staff interviewed identified the need to include more relatives and care staff in sensory activities and to use sensory facilities more frequently. However, there was some conflict to whether activities were within the remit of care staff, with some reporting this was the exclusive role of the activity co-ordinator. 
Care workers and families need to be more involved. (P019)

It shouldn't be just the activities team who are using the space with the residents. (P011)

\section{...there was some conflict to whether activities were within the remit of care staff, with some reporting this was the exclusive role of the activity co-ordinator.}

Contrary to the comments that families needed to be more involved, others felt it should be the domain of staff. Staff acknowledged that relatives might benefit from the MSE, but their inclusion in sensory activity was not always encouraged.

Happened just once (daughter of resident), has not really been encouraged by staff. (P019)

It's good it helps their relatives. (P018)

It was unclear from the participant responses to why more relatives had not made use of the sensory space with their family member, but this may have been due to families not knowing how to use the equipment or understanding how to facilitate a session. Given that staff reported a gap in their understanding in how to facilitate sensory activity, this is unsurprising.

\section{Discussion}

This study endeavored to identify sensory equipment available in care home settings, the design of sensory spaces, and implementation challenges as identified by care staff.

Key themes included the need for clearer direction in equipment selection, training in how to use it, and ongoing support to develop a sensory approach using age appropriate equipment for people with dementia. Staff were keen to learn more about how to engage people with dementia in sensory activity as well as how to design a versatile and accessible multisensory space. There was also a recognition that the design of the space and equipment should be more appropriate for older people with dementia and should address all sensory modalities.

Many of the staff reported being unsure of what the sensory room was for but did feel it was of benefit especially for those in the later stages of the disease. This ambiguity experienced by staff was also identified by others. Indeed, Anderson, Bird, MacPherson, McDonough, and Davis (2011) reported that implementation of sensory rooms was a major barrier to achieving the desired outcomes reported by other studies (Baker et al., 2001; Collier et al., 2010). Anderson noted "considerable enthusiasm" from management but lack of commitment from care staff due to "time pressure, competing work commitments and being understaffed" (Anderson et al., 2011, p. 173). These findings support the need for a cohesive unified approach both in supporting staff at all levels and in adopting a clear protocol, such as the PAL activity profile for MSEs (Pool, 2012), that is achievable given staff availability, workloads, and accessibility of the sensory environment.

Associated with the points above, staff also acknowledged the need for further training both in setting up a suitable multisensory space and in facilitating a sensory session. Where training was given, it tended to focus on how to operate the equipment rather than how to run a session. Staff perceived this as a barrier to using the sensory equipment successfully. The need for robust training and support was highlighted in a systematic review by Livingston et al. (2014). The review identified that person-centered training of care staff had the capacity to reduce symptomatic and severe agitation in residents in care homes. Secondary to this, it was found that ongoing supervision of staff following training significantly improved care/resident communication. The review also identified that sensory interventions had a significant effect on agitation in residents when staff were trained to use a sensory protocol. The benefit of staff training and ongoing supervision of staff who facilitate activities was also noted by Gitlin et al. (2008).

Staff also acknowledged the need for more age appropriate equipment to be used alongside more familiar items in order to maximize the use of the MSE. Many of the brightly colored pieces of typical MSE equipment currently offered by MSE suppliers were seen to be juvenile and not easily recognizable or familiar to the resident. A closer link between the sensory space and the everyday 
environment was also desired. The lack of a multisensory approach was also identified in the quantitative data where visual stimulation was found to be most likely to be stimulated. This was in contrast to the senses of taste, smell, proprioception, and the vestibular system, which were often underrepresented in most of the sensory rooms observed. This was an issue also reported by Baker et al. (2001) and Collier, McPherson, Ellis-Hill, Staal, and Bucks (2010) who identified that visual equipment was used predominantly in sensory spaces. It is perhaps this lack of understanding of the multisensory approach due to poor or limited training that has led to a strong focus on one or two senses but also the desire to create a facility that has a strong visual appeal to prospective residents and funders.

As a result of this study, design principles were collated and reviewed by a steering group and relevant stakeholders. A subsequent guide to multisensory design was produced (Jakob \& Collier, 2015). Key principles revolved around the need to have access to equipment that stimulates all sensory modalities with different intensities.

\section{Key principles revolved around the need to have access to equipment that stimulates all sensory modalities with different intensities.}

For example, the subtle lighting of an optic fiber through to a galaxy panel of bright lights. Sensory equipment also needs to range from highly technical to familiar and low tech. Elements of reminiscence articles selected for their sensory components were perceived to be particularly accessible for residents. For example, focusing on the sensory aspects of an activity undertaken in the past such as sorting through silk scarves was one activity identified as accessible by care staff.

\section{Elements of reminiscence articles selected for their sensory components were perceived to be particularly accessible for residents.}

Staff need basic training in both how to facilitate engagement in sensory activity and how to identify sensory preference. This training could be extended to include residents' family and friends to increase participation. Giving care staff and family members support and guidance on how to facilitate and support activity will maximize opportunities for more meaningful engagement for the person with dementia (Gitlin et al., 2008).

While this study is limited in its focus on a small number of care settings within the south of England, it will be expanded to include a wider range of care settings across the UK to further explore the concept of successful sensory design for people with dementia.

\section{Limitations}

This study did have a number of limitations including the restriction of only using care homes in the South. This may have resulted in location bias, although there was a mix of rural and innercity care homes. While the results were presented as organizational responses, it is not known if different professional groups employed within the care homes would have had particular bias toward sensory activity and design. The presence of the researchers may have also influenced responses from participants particularly in relation to participants desire to provide socially desirable responses or by adopting certain behaviors in order to be perceived as "good participants." Participants were assured anonymity, and questions from the semi-structured interview were asked in a conversational manner that appeared to relax participants. Residents with dementia were not consulted during this study but a subsequent study is underway that will further explore the experiences of multisensory approaches from the perspective of individuals with dementia.

\section{Conclusion}

Given that previous research has provided some support for the use of multisensory stimulation using MSEs and sensory spaces (Baker et al., 2001; Collier et al., 2010; Sanchez et al., 2013; van Weert et al., 2005), the findings from this study suggest that most sensory rooms and MSEs 
in care home settings are not achieving some of the proposed benefits due to a number of factors. The results suggest that many care homes do not feature an appropriate range of sensory equipment, and care staff believe many residents struggle to engage with equipment due to the highly technical features of the equipment. The principles of multisensory stimulation are often neglected with visual and tactile equipment being used as the primary stimulus, and gustatory and vestibular/proprioceptive stimulation often not being offered at all. Therefore, a multisensory experience is not available. There appears to be a lack of guidance of what to include in a sensory room/MSE for people with dementia, with many staff relying on trial and error to achieve a satisfactory outcome such as the person becoming calmer. Staff feel that they lack the skill and knowledge to set up and utilize a sensory activity to the benefit of residents and would like to expand their knowledge further. Staff believe that sensory rooms and MSEs are good for people with dementia, particularly those at the later stages of the disease. Staff would like to be able to expand this approach into general living area using more age appropriate and reminiscence sensory equipment, while being mindful of the impact of sensory stimulation on the engagement of other residents within the home.

\section{Implications for Practice}

- Multisensory design should be considered in all areas of the care home environment. Design should incorporate stimulation of all senses including sight, sound, touch, taste, smell, and movement.

- Barriers to engagement with MSEs include lack of appropriate training of staff in assessment and activity facilitation and finding a suitable space for a sensory area. A clearer direction in equipment selection, training on how to use equipment, and ongoing staff support is required.

- Sensory spaces should be considered in all areas of the care home setting. This equipment may be related to the different areas of the home such as sensory bath products and seashore items in the bathroom.

- Equipment development should include age appropriate methods of delivering sensory stimulation. There is a need for sensory spaces that include both hi-tech and lowtech solutions, new and familiar equipment.

- Further research is required to identify the benefits achieved by using a multisensory approach in a care home setting with an aspect exploring the experiences of people with dementia.

\section{Declaration of Conflicting Interests}

The authors declared no potential conflicts of interest with respect to the research, authorship, and/or publication of this article.

\section{Funding}

The authors disclosed receipt of the following financial support for the research, authorship, and/or publication of this article: This work was supported by the Arts and Humanities Research Council (AHRC) under grant nos. AH/K003135/1.

\section{References}

Anderson, K., Bird, M., MacPherson, S., McDonough, D., \& Davis, T. (2011). Findings from a pilot investigation of the effectiveness of a Snoezelen room in residential care: Should we be engaging with our residents more? Geriatric Nursing, 32, 166-176.

Ayres, A. (1972). Sensory integration and learning disorders. Los Angeles, CA: Western Psychological Services.

Baker, R., Bell, S., Holloway, J., Pearce, R., Dowling, Z., Thomas, P., ... Wareing, L. A. (2001). A randomized controlled trial of the effects of multi-sensory stimulation (MSS) for people with dementia. British Journal of Clinical Psychology, 40, 81-96.

Baker, R., Dowling, Z., Wareing, L.-A., Dawson, J., \& Assey, J. (1997). Snoezelen: Its long-term and short-term effects on older people with dementia. British Journal of Occupational Therapy, 60, 213-218.

Bauer, M., Rayner, J. A., Koch, S., \& Chenco, C. (2012). The use of multisensory interventions to manage dementia-related behaviours in the 
residential aged care setting: A survey of one Australian state. Journal of Clinical Nursing, 21, 3061-3069.

Braun, V., \& Clarke, V. (2006). Using thematic analysis in psychology. Qualitative Research in Psychology, 3, 77-101.

Collier, L. (2012). Chapter 9: Planning and implementing sensory interventions. In J. Pool (Ed.), The pool activity level (PAL) instrument for occupational profiling (pp. 140-153). London, England: Jessica Kingsley.

Collier, L., McPherson, K., Ellis-Hill, C., Staal, J., \& Bucks, R. (2010). Multisensory stimulation to improve functional performance in moderate to severe dementia-interim results. American Journal of Alzheimer's Disease and Other Dementias, 25, 698-703.

Dalke, H., \& Corso, A. (2010). Living with dementia: Can design make a difference? London, England: Kingston University.

Fleming, R., \& Bennett, K. (2015). Assessing the quality of environmental design of nursing homes for people with dementia: Development of a new tool. Australasian Journal on Ageing, 34, 191-194.

Garre-Olmo, J., Lopez-Pousa, S., Turon-Estrada, A., Juvinya, D., Ballester, D., \& Vilalta-Franch, J. (2012). Environmental determinants of quality of life in nursing home residents with severe dementia. Journal of the American Geriatrics Society, 60, 1230-1236.

Gitlin, L., Winter, L., Burke, J., Chernett, N., Dennis, M., \& Hauck, W. (2008). Tailored activities to manage neuropsychiatric behaviours in persons with dementia and reduced caregiver burden: A randomised pilot study. American Journal of Geriatric Psychiatry, 16, 229-239.

Hogg, J., Cavet, J., Lambe, L., \& Smeddle, M. (2001). The use of 'Snoezelen' as multisensory stimulation with people with intellectual disabilities: A review of the research. Research in Developmental Disabilities, 22, 353-372.

Hulsegge, J., \& Verheul, A. (1987). Snoezelen. Chesterfield, England: Rompa.

Jakob, A., \& Collier, L. (2015). How to make a sensory room for people living with dementia. Unpublished manuscript, Kingston University, England.

Kolanowski, A., Litaker, M., \& Buettner, L. (2005). Efficacy of theory based activities for behavioural symptoms of dementia. Nursing Research, 54, 219-228.
Kovach, C. R. (2000). Sensoristasis and imbalance in persons with dementia. Journal of Nursing Scholarship, 32, 379-384.

Leone, E., Deudon, A., Piano, J., Robert, P., \& Dechamps, A. (2012). Are dementia patient's engagement using tailored stimuli the same? The apathy dilemma in nursing home residents. Current Gerontology and Geriatrics Research, 2012, 11. Retrieved from http://dx.doi.org/10.1155/2012/ 942640.

Livingston, G., Kelly, L., Lewis-Holmes, E., Baio, G., Morris, S., Patel, N., ... Cooper, C. (2014). Nonpharmacological interventions for agitation in dementia: Systematic review of randomised controlled trials. British Journal of Psychiatry, 205, 436-442.

Lykkeslet, E., Gjengedal, E., Skrondal, T., \& MayBritt, S. (2014). Sensory stimulation-A way of creating mutual relations in dementia care. Qualitative Studies on Health and Well-being, 9. doi:10. 3402/qhw.v9.23888

Maseda, A., Sanchez, A., Marante, M. P., GonzalezAbraldes, I., de Labra, C., \& Millan-Calenti, J. C. (2014). Multi-sensory stimulation on mood, behavior, and biomedical parameters in people with dementia: Is it more effective than conventional one-to-one stimulation? American Journal of Alzheimer's Disease and other Dementias, 29, 637-647.

Moffat, N., Barker, P., Pinkney, L., Garside, M., \& Freeman, C. (1993). Snoezelen an experience for people with dementia. Chesterfield, England: Rompa.

Moore, K. J., Hill, K. D., Robinson, A. L., Haynes, T. P., Haralambous, B., \& Nitz, J. C. (2011). The state of physical environments in Australian residential aged care facilities. Australian Health Review, 35, 412-417.

National Institute for Health and Care Excellence. (2006). Dementia: Supporting people with dementia and their carers in health and social care (NICE guideline CG42). London, England: National Institute for Clinical Excellence.

Patton, M. Q. (2002). Qualitative research \& evaluation methods (3rd ed.). Thousand Oaks, CA: Sage.

Polit, D., \& Beck, C. (2013). Essentials of nursing research: Appraising evidence for nursing practice. Philadelphia, PA: Lippincott Williams \& Wilkins.

Pool, J. (2012). The Pool Activity Level (PAL) instrument for occupational profiling. London, England: Jessica Kingsley. 
Riley-Doucet, C. K., \& Dunn, K. S. (2013). Using multi-sensory technology to create a therapeutic environment for people with dementia in an adult day center: A pilot study. Research on Gerontological Nursing, 6, 225-233.

Sanchez, A., Millan-Calenti, J., Lorenzo-Lopez, L., \& Maseda, A. (2013). Multisensory stimulation for people with dementia: A review of the literature. American Journal of Alzheimer's Disease and Other Dementia, 28, 7-14.

Schaaf, R. C., \& Miller, L. J. (2005). Occupational therapy using a sensory integrative approach for children with developmental disabilities. Mental Retardation and Developmental Disabilities Research Reviews, 11, 143-148.
Scholfield, P. (1996). Snoezelen: Its potential for people with chronic pain. Complementary Therapy in Nursing and Midwifery, 2, 9-12.

Staal, J., Pinkney, L., \& Roane, D. (2003). Assessment of stimulus preferences in multi-sensory environment therapy for older people with dementia. British Journal of Occupational Therapy, 66, 542-550.

StrØmb, B., Ytrehus, S., \& Ellen-Karine, G. (2016). Sensory stimulation for people with dementia: A review of the literature. Journal of Clinical Nursing, 25, 1805-1834.

van Weert, J. C., van Dulmen, A. M., Spreeuwenberg, P. M., Bensing, J. M., \& Ribbe, M. W. (2005). The effects of the implementation of Snoezelen on the quality of working life in psychogeriatric care. International Psychogeriatrics, 17, 407-442. 